\title{
Turning over a new leaf: the Aspen Spatial Bibliography enhances research
}

\author{
Anne Hedrich, Andrea Payant, Liz Woolcott, Steven Petersen, Ryan Howell and \\ Paul C. Rogers
}

\section{Introduction}

Librarians have long provided bibliographic access to materials. Organization for access, based on information facets such as author and title, has been widely successful. Where it can fall short, though, is for spatiallyor location-based information, as well as for patrons inclined to visual or spatial cognition. A spatial basis for access can meet this need.

Using traditional bibliographic databases (and paper indexes and abstracts) to find information that is place-based can be cumbersome. Locations can be described by naming natural features such as mountains and lakes or by political demarcations such as states and countries. Research in an area may be described on a scale ranging from the very local to a global region, and this scale of a research locale may or may not be addressed in a helpful way in geographic fields (if those fields are present at all in a database or in controlled vocabulary such as subject headings). Useful resources might be missed in a database search because place names can vary between academic and local naming, over time and among cultures and languages. Sometimes the best a researcher can hope for is to narrow the search results down to a more manageable number to look through, but there remains the chance of missing relevant sources because of the ambiguous nature of using words to describe place. Matching the authordescribed scale and terminology can be a matter of luck.

The Aspen Spatial Bibliography was created to provide spatial searching options for access to literature in quaking aspen sciences (e.g. Populus tremuloides). Housed at Brigham
Young University's Geospatial Habitat Analysis Lab, and also accessible by this link https://arcg.is/mDPCi0, it is a bibliography expressed as an interactive map that displays and allows you to search geographically within the subject of aspen research.

Geographical display and visualization is increasingly appearing in Digital Humanities activity, for example, Eskin (2018) writes of having students explore texts in English literature by placing them geographically using the StoryMap product. Bibliographies are also created for a locale and placed on a map, such as the Coos Bay Bibliography (Schmitt and Butler, 2012). The Aspen Spatial Bibliography application uses geographic information system (GIS) technology to geographically place a scientific subject that has a spatial component to highlight and allow search by its geographic components or geographic scope of the research.

\section{Structure}

The Aspen Spatial Bibliography resource is derived from an extensive traditional bibliography, the Aspen Bibliography, of nearly 6,000 aspen science resources, primarily research articles but also reports, dissertations and theses, and even a computer program. The Aspen Bibliography was created by David Burton of the Aspen Delineation Project and was then picked up by (article author) Dr Paul Rogers of the Western Aspen Alliance, who continues to add to it. As a standard format bibliography, it is housed in the Institutional Repository at USU Libraries https://digitalcommons.usu. edu/aspen_bib/, at Utah State University in Logan, UT. Researchers Dr Steven Petersen and Ryan Howell at
Brigham Young University approached Dr Rogers in 2015 about the prospect of making a geographic interface for the aspen bibliography.

The Aspen Spatial Bibliography was created using Esri's ArGIS Online. Each item in the bibliography is expressed as a point on the map. Item records have three types of fields: citation, access (pointers to full text) and geographic. Basic citation information is provided: author, title, publication, publication date and pages. (The working group is exploring bringing in keywords and abstracts.) Item access fields provide pointers to full text such as DOIs, as well as links to the Aspen Bibliography record, which provides full text, links to full text or a link to a WorldCat record that can assist in finding the item in a local library. Geographic fields are used to place the item on the map. Coordinates are expressed in Latitude/Longitude Decimal Degrees. Coordinates are described as:

Provided (coordinates are given by authors),

Estimated (location description, such as Bald Mountain, or near Shawano, Wisconsin, or in Chippewa National Forest, is provided by authors, but coordinates are not given),

Generalized (location is listed in a paper in general terms, such as northern Utah)

Not Applicable (research done in a lab or the item is something like a literature review)

To create the interactive map, coordinates and coordinate type, as well as citation information, such as author, title, year, and so on, were assigned in a point feature class in a geodatabase (Figure 1). Using ArcGIS pro, that file 
Figure 1. Initial screen of the Aspen Spatial Bibliography

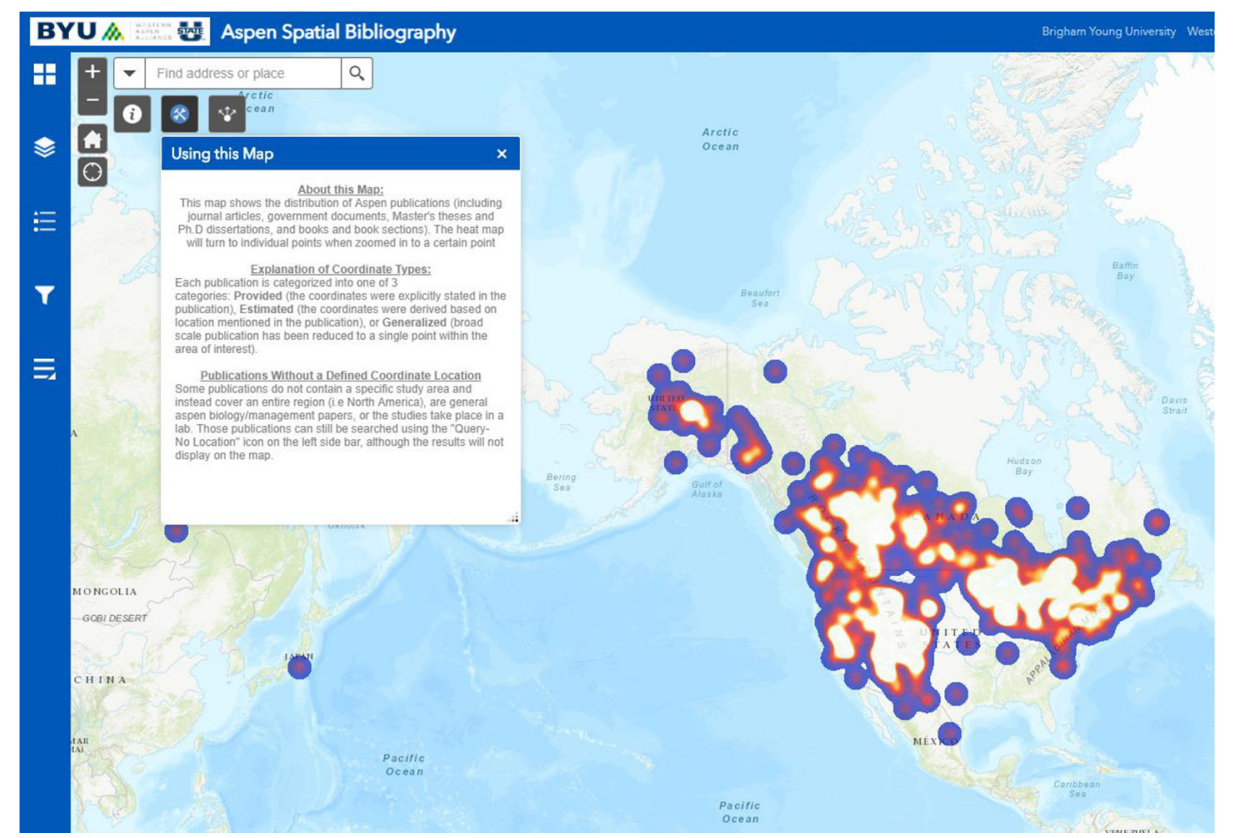

was uploaded to ArcGIS online as a hosted layer. A Web map was created to display the point file. A copy of the point file was also displayed as a heat map. Visibility limitations were set so that the heat map is visible from a small scale (showing a large area) while the individual point layer is visible from a larger scale (zoomed in). Widgets were added using ArcGIS Online available widgets to improve the user interface, including features to change the basemap and to create queries.

The Aspen Spatial Bibliography is a work in progress. Approximately one third of the Aspen Bibliography records are in the spatial bibliography. Processes for entering data into the GIS have been tweaked, as more efficient methods are developed, but it remains time-intensive to find the full text of each item in the bibliography and determine the location of the research. Adjustments to the Web map interface have also been made to improve display and query (search) functionality.

\section{Searching and using the Aspen Spatial Bibliography}

Each item in the bibliography is a dot on the map. Concentrations of dots show as a heat map (warmer colors for areas of high concentrations of dots/research studies) when view is zoomed out, and the dots become distinct as the focus is narrowed. Clicking on a dot brings up a small screen showing citation information and access information (links to the Aspen Bibliography record and DOI if available).

Searching can be conducted by panning the map to move the view, zooming in and out of areas and drawing a boundary of an area to focus on. An attribute table can be called up that is populated with items for the geographic area that is displayed or delineated (Figure 2).

There is a bibliographic search interface in the Aspen Spatial Bibliography as well, which allows querying and filtering in typical citation fields such as author and title and keyword. Results appear as points on the map, and query results also show a list of items along the side of the screen (Figures 3 and 4).

\section{Using geography to explore research literature}

There are many reasons why a researcher might want to search spatially for information. Researchers may want to know what kind of research has been done on aspen in a specific geographic area. A topic within aspen research can be mapped out for geographic trends, such as the fire and aspen example shown in Figure 5. It can be interesting to find out, more broadly, where research has been done and compare it to where aspen grows (there is an aspen extent layer that can be activated on the map) and conversely to see areas where there is no research. Researchers may be looking for locations where no research within a given topic in aspen science has been done for comparative studies. A search can quickly show where a researcher works and who works in certain areas for possible collaboration. It might also be of interest to see how research in a geographic area or on a topical interface with location has changed over time. Finally, it may be of interest to know if research on specific aspen topics has yielded differing or concurring results based on locale.

Research trends may show up at different scales, ranging from the global to a place smaller than an acre. The ability to easily zoom in and out to change the geographic extent of the search and the result list makes this a quick exercise, rather than coming up with the many terms that might be needed to make a successful and comprehensive search in a bibliographic database. 
Figure 2. Item information display

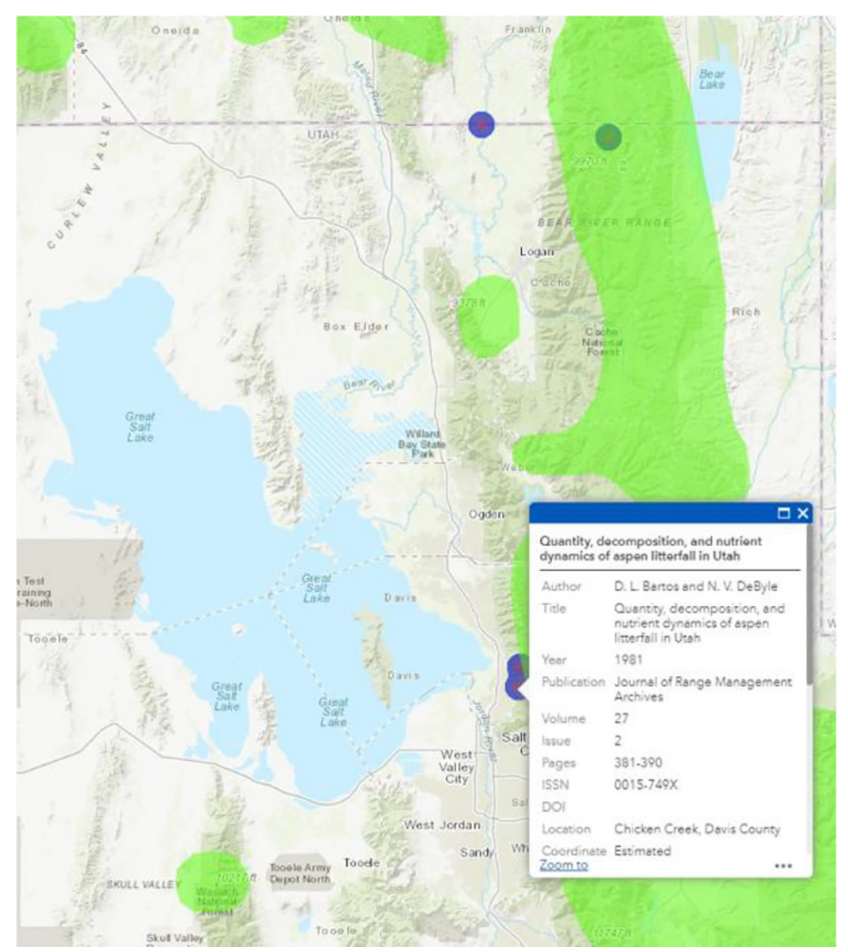

Figure 3. Aspen Spatial Bibliography bibliographic search interface

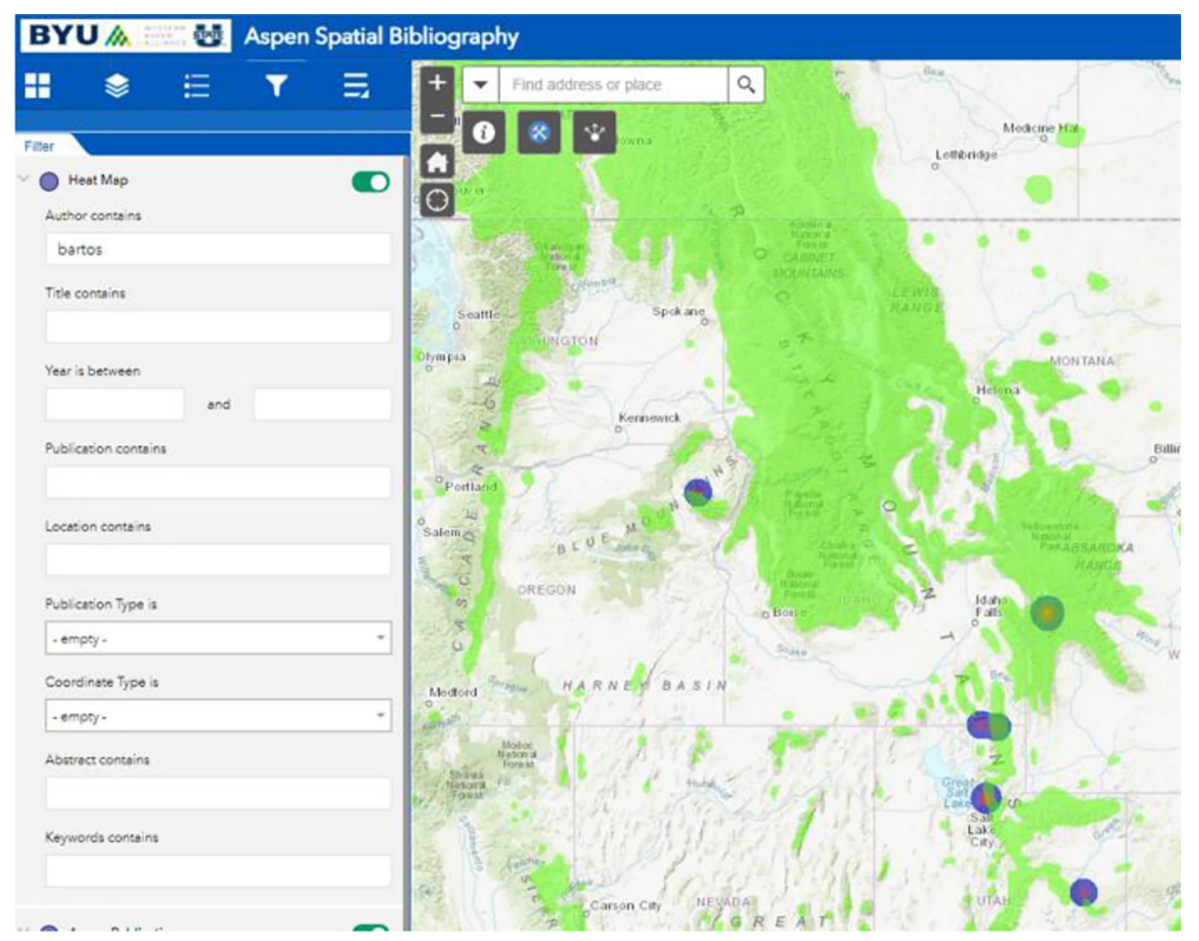

\section{Collaborations}

The Aspen Spatial Bibliography is a product of collaboration between institutions (Brigham Young University and Utah State University) and between researchers, GIS technicians and librarians. It illustrates how librarians can work with subject area experts to create useful new tools for access to information, and how extending outside of our usual institutional and professional boundaries can use the strengths of the different participants to bring innovative ideas to practical fruition.

Researchers Rogers, Petersen, and Howell have the subject area background and provide the GIS know-how in setting up the resource. Librarians Hedrich, Payant and Woolcott provide guidance on descriptive metadata and accessibility. Specific librarian contributions have included the creation of access points leading to full text resources and standardizing terms for item type. For example, librarians advised on the inclusion and use of bibliographic information such as keywords and abstracts in the resource records.

\section{Branching out}

Libraries are increasingly teaching data visualization techniques, including spatial display, as a service, and incorporating spatial display in featuring library resources. Geographic bibliographies can provide opportunities for libraries and organizations to showcase unique collections that have a spatial component, such as theses and dissertations and materials in special collections.

While the Aspen Spatial Bibliography illustrates a demonstration of these concepts, it is easy to envision many subject areas, particularly in the natural and social sciences, where space-place attributes may be of great interest.

\section{Conclusion}

The Aspen Spatial Bibliography provides a unique, valuable tool for aspen researchers. Geographic representation of a large body of knowledge of aspen science provides new insight into this field of study. The capability to search geographically for aspen research offers new opportunities for discovery of information, uncovers connections 
Figure 4. Keyword search results displayed bibliographically and geographically

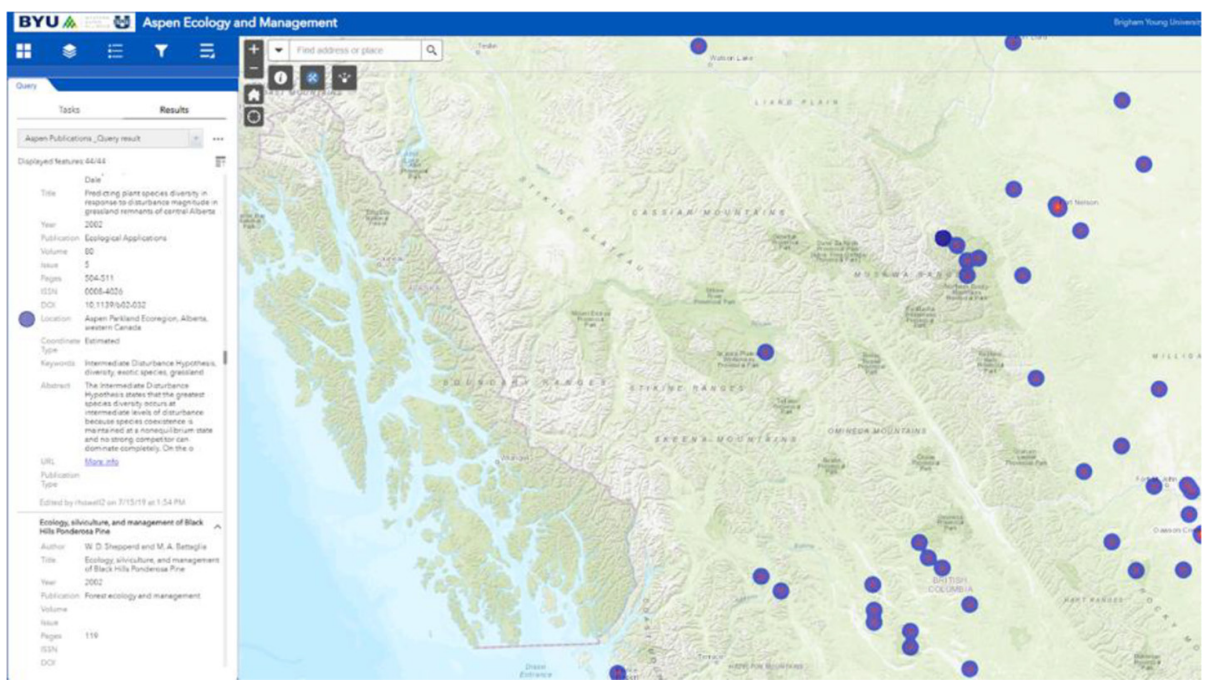

Figure 5. Map display shows at a glance that aspen research with a focus on fire has been done in North America but not in Europe

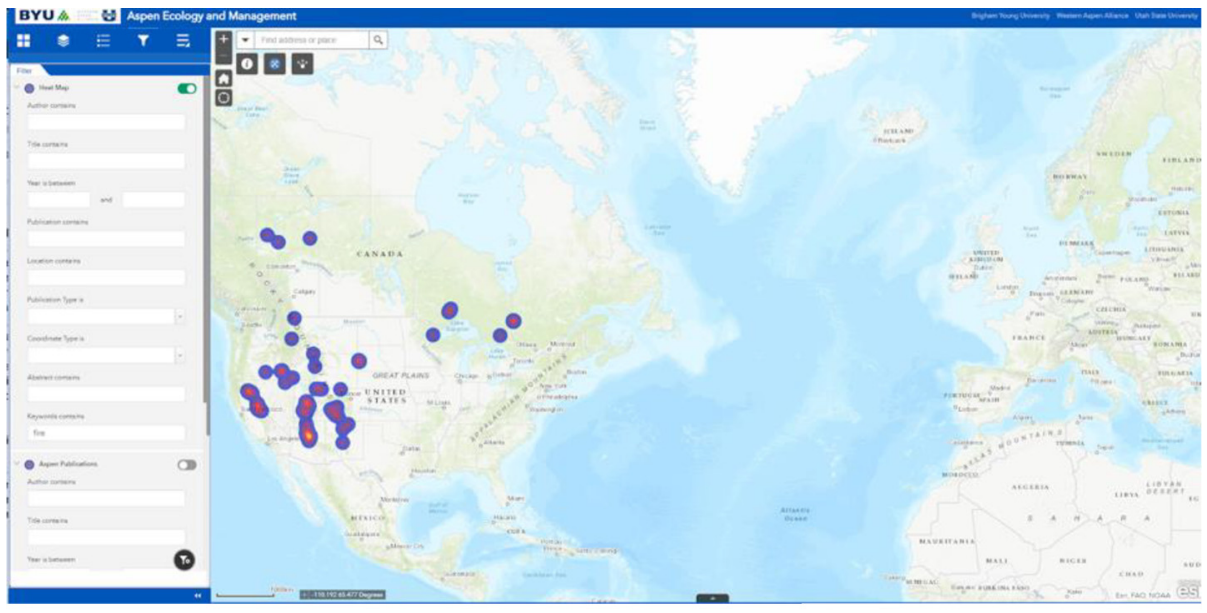

Note: This search may display differently as more studies are entered into the Aspen Spatial Bibliography

between studies and fosters collaboration between researchers.

Further, this technique of bringing spatially-based science research information into an interactive map can be applied to a number of different subject areas. Using tools such as ArcGIS Online that offer geographic searching, librarians can open up a whole new world (pun intended) of access to research and information that has a spatial component.

\section{Funding}

Paul C. Rogers was supported by the Western Aspen Alliance Donation Fund, EJF Philanthropies, and the US Bureau of Land Management (L16AC00175).

\section{REFERENCES}

Eskin, C.R. (2018), "Books are not absolutely dead things': English literature, material culture and mapping text", International Journal of Humanities and Arts Computing, Vol. 12 No. 1, pp. 37-47.

Schmitt, J. and Butler, B. (2012), "Creating a geo-referenced bibliography with Google 
earth and GeoCommons: the coos bay bibliography", Issues in Science \& Technology Librarianship, No. 71, pp. 1-1, doi: 10.5062/F43J39XH.

Anne Hedrich (anne.hedrich@usu. edu), Andrea Payant (andrea.payant@ usu.edu), and Liz Woolcott (liz. woolcott@usu.edu) are based at USU
Libraries, Utah State University, Logan, Utah, USA.

Steven Petersen (steven petersen@ byu.edu) is based at Forest and Rangeland Landscape Ecology, GIS, and Remote Sensing, Brigham Young University, Provo, Utah, USA.

Ryan Howell (howell.ryan12@ gmail.com) is based at the Department of Plant and Wildlife Sciences, Brigham Young University, Provo, Utah, USA.

Paul C.Rogers (p.rogers@usu.edu) is based at the Department of Environment and Society and the Ecology Center, Utah State University, Logan, Utah, USA. 